\title{
ENHANCED CLOUD DISRUPTION BY MAGNETIC FIELD INTERACTION ${ }^{1}$
}

\author{
G. Gregori ${ }^{2,3}$ Francesco Miniati,${ }^{3}$ Dongsu Ryu, ${ }^{4}$ and T. W. Jones ${ }^{3}$ \\ Received 1999 August 8; accepted 1999 October 18; published 1999 November 22
}

\begin{abstract}
We present results from the first three-dimensional numerical simulations of moderately supersonic cloud motion through a tenuous, magnetized medium. We show that the interaction of the cloud with a magnetic field perpendicular to its motion has a great dynamical impact on the development of instabilities at the cloud surface. Even for initially spherical clouds, magnetic field lines become trapped in surface deformations and undergo stretching. The consequent field amplification that occurs there and, in particular, its variation across the cloud face then dramatically enhance the growth rate of Rayleigh-Taylor unstable modes, hastening the cloud disruption.
\end{abstract}

Subject headings: instabilities - ISM: clouds — ISM: kinematics and dynamics — methods: numerical — MHD

\section{INTRODUCTION}

This Letter focuses on the results of the first three-dimensional study of the ballistic interaction of a moderately supersonic dense cloud with a warmer magnetized tenuous medium. Magnetic fields are ubiquitous in astrophysical environments and cannot be neglected in any realistic interstellar medium-related study. Mac Low, McKee, \& Klein (1994) and Jones, Ryu, \& Tregillis (1996) have studied the two-dimensional magnetohydrodynamics (MHD) of cosmic bullets, extending earlier gasdynamical simulations and addressing the importance of the magnetic field in preventing the cloud disruption due to fluid instabilities. In particular, Jones et al. (1996) found that a region of strong magnetic pressure (or a magnetic bumper) develops at the nose of the cloud during its motion through a magnetized medium, whenever its velocity is perpendicular to the initial unperturbed background field (transverse case). This enhancement was attributed to a strong stretching of the magnetic field lines. Miniati, Jones, \& Ryu (1999a) investigated the problem further, pointing out that the behavior observed in the transverse case was typical, except in the case of a very small angle $\left(<30^{\circ}\right)$ between the cloud velocity and the initial magnetic field. In addition, the development of this magnetic shield in front of the cloud was recognized as playing a crucial role in the outcome of cloud collisions (Miniati et al. 1999b) because it dramatically reduced the degree of cloud disruption. Furthermore, it was important in terms of the exchange of magnetic and kinetic energy between different phases in the interstellar medium (ISM; Miniati et al. 1999a) and in highlighting cosmic-ray electrons (Jones, Kang, \& Tregillis 1994).

While supersonic motion of an individual cloud is obviously idealized, there are numerous astronomical contexts that can be illuminated by its example. For example, MHD wind-cloud interaction may be important for the evolution of synchrotron emission processes from planetary nebulae (Dgani \& Soker 1998). Recently, a multiphase structure has been proposed for the dynamical state of the cosmic intracluster medium (Fabian

\footnotetext{
${ }^{1}$ Animations and color images from this work have been posted at http:// www.msi.umn.edu/Projects/twj/mhd3d/.

${ }^{2}$ Department of Mechanical Engineering, University of Minnesota, Minneapolis, MN 55455; gregori@me.umn.edu.

${ }^{3}$ School of Physics and Astronomy, University of Minnesota, Minneapolis, MN 55455; min@msi.umn.edu, twj@astro.spa.umn.edu.

${ }^{4}$ Department of Astronomy and Space Science, Chungnam National University, Daejeon, 305-764, Korea; ryu@canopus.chungnam.ac.kr.
}

1997), in order to explain EUV radiation in excess to what is expected from the hot gas there (e.g., Bowyer, Lieu, \& Mittaz 1998). Although the issue has not been settled yet, this suggestion reinforces the necessity for understanding the proper evolution of clouds in a multiphase medium.

All the previous MHD cloud-motion results were based on two-dimensional numerical simulations, so the importance of following them up with more realistic three-dimensional calculations is clear. J. M. Stone and collaborators (Stone \& Norman 1992; Xu \& Stone 1995) have reported three-dimensional hydrodynamical simulations of shocked gas clouds. They found qualitative agreement with analogous two-dimensional gasdynamical simulations. However, as we shall see, the introduction of nonisotropic Maxwell stresses produces very significant effects that differ in three dimensions from what has been seen in previous two-dimensional MHD simulations.

\section{NUMERICS AND PROBLEM SETUP}

The numerical computation is based on a total variation diminishing (TVD) scheme for ideal MHD (Ryu \& Jones 1995; Ryu, Jones, \& Frank 1995; Ryu et al. 1998). The cloud, initially spherical, is set in motion with respect to the uniform ambient medium with a velocity aligned along the $x$-axis. Its velocity is $u_{c}=M c_{s}$, where $c_{s}$ is the sound speed in the ambient medium, and the Mach number is $M=1.5$. The initial cloud density is $\rho_{c}=\chi \rho_{i}$, with $\rho_{i}$ being the intercloud density and $\chi=100$. The direction of the magnetic field is chosen along the $y$-axis. Its intensity is conveniently expressed in terms of the familiar parameter $\beta=p / p_{B}$, where $p$ is the hydrodynamic pressure and $p_{B}=B^{2} /(8 \pi)$ is the magnetic pressure. In these numerical simulations, we have considered the cases of both a strong field $(\beta=4)$ and a weak field $(\beta=100)$. To be able to compare them with pure hydrodynamic effects, a case with $\beta=\infty$ (no magnetic field) has also been computed. The calculations have been performed using a $416 \times 208 \times 416$ zone box containing onequarter of the physical space with radial symmetry applied in the $y$ - $z$ plane around the $x$-axis. The initial cloud radius spanned 26 zones. Additional details on the numerical setup can be found in a companion paper describing quantitative results (G. Gregori, F. Miniati, D. Ryu, \& T. W. Jones 1999, in preparation). Since the cloud motion is supersonic, its motion leads to the formation of a forward bow shock and a reverse crushing shock propagating through the cloud. The approximate time for the latter to cross the cloud is referred to as the "crushing 


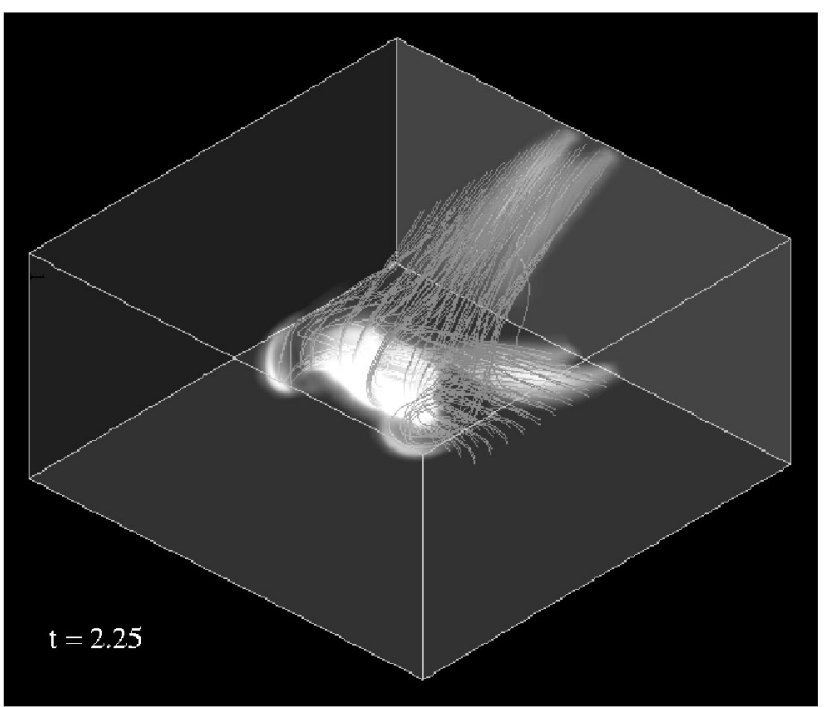

FIG. 1.-Volume rendering (log scale) of the magnetic pressure for the $\beta=4$ simulation (strong field) at $t=2.25 \tau_{\mathrm{cr}}$. The magnetic field lines are superposed in the figure.

time",5 (e.g., Jones et al. 1994): $\tau_{\mathrm{cr}}=2 R_{c} \chi^{1 / 2} / M c_{s}$, where $R_{c}$ is the initial cloud radius. As we will see, the crushing time is a relevant dynamical quantity since it is proportional to the time over which disruptive instabilities develop.

\section{CLOUD DISRUPTION}

As the cloud moves through the ISM, its surface is subjected to several instability mechanisms: namely, the KelvinHelmholtz (K-H), the Rayleigh-Taylor (R-T), and, at start-up, the Richtmyer-Meshkov (R-M) instability. These instabilities, but especially the R-T instability, will ultimately disrupt the entire cloud. Grid-scale noise provides the seed for initial development of such instabilities (Kane, Drake, \& Remington 1999 and references therein). At the same time, finite numerical resolution may suppress the large wavenumber (small-scale) mode components that have the fastest initial growth rate (Chandrasekhar 1961). The K-H instability is related to the shearing motion at the separation between two fluids. Typically, we should expect such an instability to develop on the lateral boundaries of the cloud. From our simulations, we can see that its effects are generally limited, especially considering that, in the MHD case, the development of the instability tends to be suppressed by the magnetic field for wavevectors in the $x-y$ plane (Chandrasekhar 1961; Jones et al. 1996). The most disruptive instability for a supersonic cloud is the R-T. It develops at the interface between two fluids when the lighter fluid accelerates the heavier one. In the limiting case of an impulsive acceleration, as at the beginning of our simulations where the clouds are set into instantaneous motion, the R-M instability applies. However, the linear growth of the R-M instability and the presence of a thin boundary layer on the initial cloud make the R-M instability relatively unimportant. Before a time $\lesssim \tau_{\text {cr }}$, the cloud interface remains R-T stable, since there is no ongoing acceleration of the cloud body to drive this instability. When

\footnotetext{
${ }^{5}$ This form of the crushing time differs from the one in Klein et al. (1994) by a factor of 2 since our definition is based on the cloud diameter instead of the cloud radius. We use this definition since it more closely measures the actual time before the crushing shock emerges.
}

the crushing shock exits the cloud, however, a pressure gradient between the front and the rear is established. The cloud body is then decelerated, and this induces the R-T growth at its front interface (e.g., Mac Low et al. 1994; Jones et al. 1994; Kane et al. 1999).

In the general hydrodynamic case, an R-T perturbation of wavenumber $k$ grows with a characteristic time $\tau_{\mathrm{RT}} \simeq$ $(g k)^{-1 / 2}$, where $g$ is the cloud deceleration (Chandrasekhar 1961). Following Klein, McKee, \& Colella (1994), we can estimate the deceleration as

$$
g \simeq \frac{\rho_{i} u_{c}^{2}}{\rho_{c} R_{c}}
$$

with the cloud velocity $u_{c}$ and the intercloud medium assumed to be at rest. It is easy to show that the most destructive modes, those with the largest wavelength, $k \sim 2 \pi / R_{c}$, develop on a timescale $\tau_{\mathrm{RT}} \sim \tau_{\mathrm{cr}}$. Thus, in the absence of magnetic field influences, the cloud will be disrupted only on a timescale of several $\tau_{\text {cr }}$ (Jones et al. 1996; Xu \& Stone 1995).

\section{RESULTS}

It is usually pointed out in the literature that in the interaction of a cloudlike object with a magnetized wind, the stretching of magnetic field lines draping around the body of the cloud is limited by their ability to escape by slipping around it (e.g., Mac Low et al. 1994; Dgani \& Soker 1998). On the contrary, our results show that the magnetic field influences the development of the cloud deformations produced by instabilities so that the field lines become trapped in such deformations. This trapping of the field lines is indeed the most important physical process that has been revealed from our three-dimensional simulations. Its main consequence is then the development of a region of strong magnetic pressure at the leading edge of the cloud. This is clearly visible in Figure 1, which shows that even at the end of the simulation, most of the "overrun" field lines are indeed kept within the R-T fingers without slipping away.

This behavior is different from what is seen at the Earth's magnetopause or from what was predicted for the magnetopause of comets (e.g., Yi et al. 1996). There, field lines do seem to slip past the object, so that it behaves somewhat like a rigid body. Indeed, for both of those cases, the impacted body is stiff because compression produces an increasingly greater restoring force.

In Figure 2, we compare the cloud evolution for the three studied cases of the field intensity through three-dimensional volume-rendering images of gas density. The four pictures in the left, middle, and right columns correspond to different evolutionary times for the progression of cases: $\beta=\infty, 100$, and 4 , respectively. The most significant conclusion is that the presence of a strong background magnetic field $(\beta=4)$ dramatically modifies the dynamical evolution of the cloud. For the hydrodynamic simulation, the cloud is initially uniformly crushed in the direction aligned with its motion, and the disruptive instabilities, which are radially symmetric because of the imposed symmetry, become evident only by the end of the simulation $\left(t=2.25 \tau_{\text {cr }}\right)$. Our resolution is not sufficient to allow really fine $\mathrm{K}-\mathrm{H}$ instabilities to develop on the cloud perimeter such as those evident in the hydrodynamical shocked-cloud results of Xu \& Stone (1995), but the global evolutions in our hydrodynamic simulations are comparable. In contrast to this behavior, the presence of a strong field ( $\beta=4$ case) causes the 

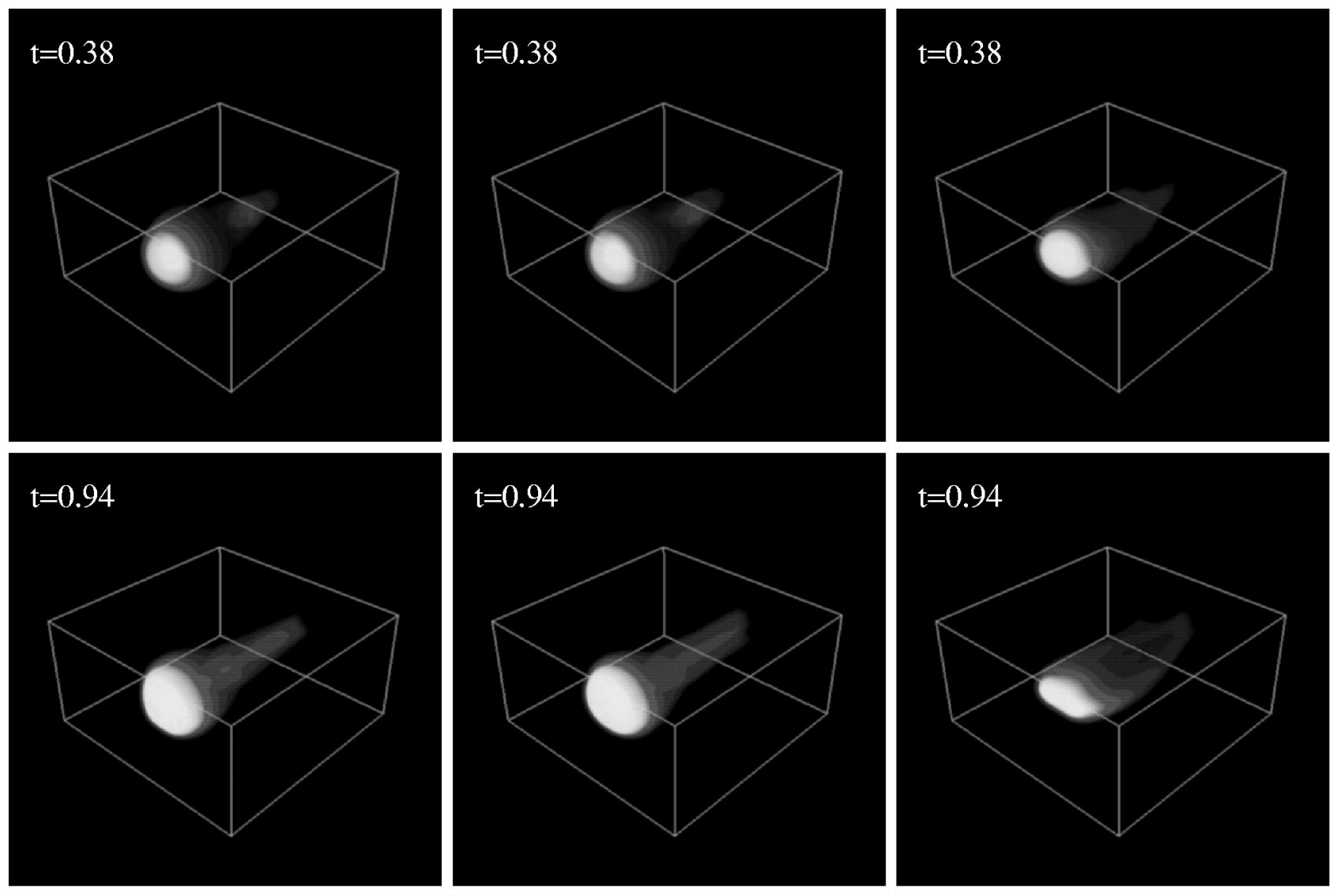

$$
\mathrm{t}=0.94
$$

$$
t=1.69
$$
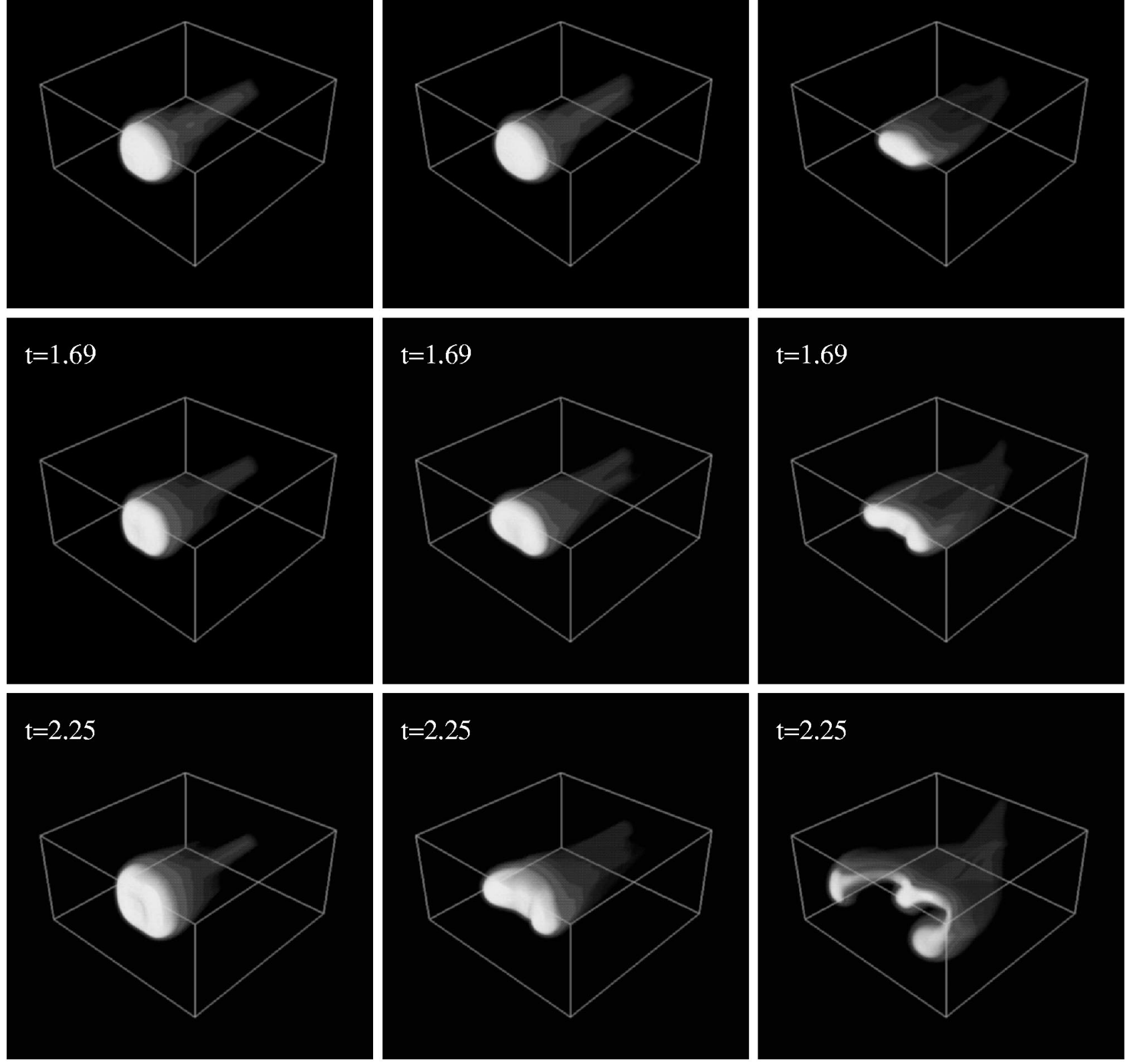

$$
t=1.69
$$

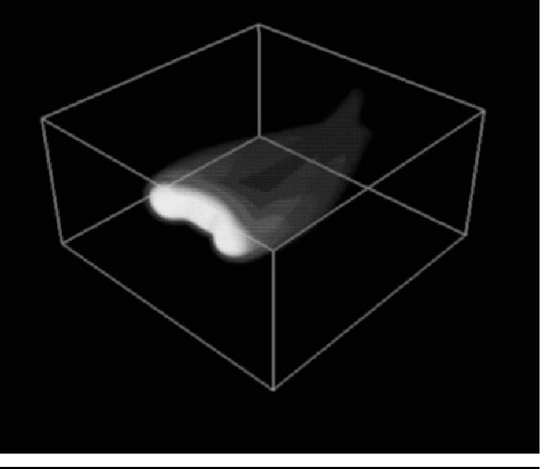

$$
\mathrm{t}=2.25
$$
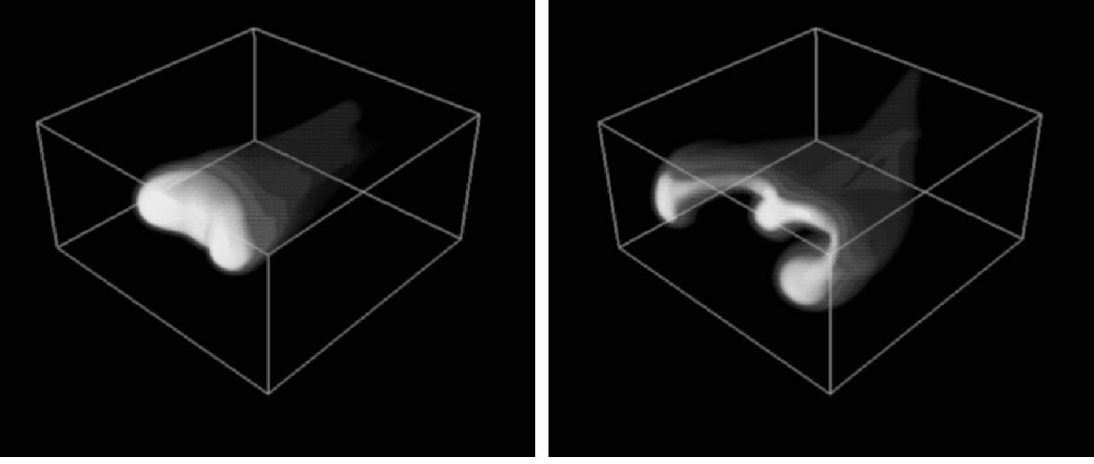

FIG. 2.-Volume rendering of the cloud density (log scale) for the $\beta=\infty$ simulation (left column of panels), $\beta=100$ (middle column of panels), and $\beta=4$ (right column of panels). Time is expressed in units of $\tau_{\mathrm{cr}}$. 
cloud to be additionally compressed along the $y$-axis by the draping magnetic field lines. As a result, the cloud is "extruded" in a direction orthogonal to the plane containing its motion and the initial field. This behavior is already clearly visible after one crushing time.

As noted earlier, magnetic fields inhibit the R-T instabilities in the $x-y$ plane (Jones et al. 1996). The development of this instability only along the $z$-axis then produces the $\mathrm{C}$-shaped structure of the cloud (last panel in the right-hand column of Fig. 2), as opposed to the symmetrical "sombrero" surface of the hydrodynamical case. In addition, the presence of the magnetic field enhances the growth of the R-T instability. In the strong-field case $(\beta=4)$, this can be assessed by replacing the ram pressure $\rho_{i} u_{c}^{2}$ with an effective pressure $\rho_{i} u_{c}^{2}+p_{B}$ in equation (1). This is motivated by the fact that, for small $\beta, p_{B}$ becomes comparable to or larger than the ram pressure $\rho_{i} u_{c}^{2}$ during times $t \lesssim \tau_{\mathrm{cr}} \sim \tau_{\mathrm{RT}}$ (see also Miniati et al. 1999a). Taking into account this additional magnetic term then shortens the timescale for the development of the instability by a factor of $\sim 2$. The instability enters the nonlinear stage sooner, and its exponential growth determines the dramatic differences from the hydrodynamical case as shown in Figure 2. In this sense, our results describe the cloud disruption by a magnetically enhanced $R$-T-like instability.

A weak field $(\beta=100)$ changes the cloud dynamics in a similar way, but more slowly and less dramatically. With a limited growth of C-shaped structures, the development of the R-T instability in front of the cloud looks typical, although it still shows evidence of the asymmetric Maxwell stresses. Also, at $t \simeq \tau_{\text {cr }}$, the magnetic pressure is still a small fraction of the ram pressure in front of the cloud. Therefore, although the shape of the cloud undergoes some deformation compared with the hydrodynamical case, the growth rate of the instability has not been enhanced significantly. Further discussions on the magnetic energy evolution and its effect on the cloud morphology can be found in G. Gregori et al. (1999, in preparation).

\section{SUMMARY}

In this Letter, we have presented the first results of a series of three-dimensional MHD numerical simulations of cloud mo- tion in a multiphase interstellar medium. We have considered a spherical cloud that moves transverse to the magnetic field, with two different cases for its initial strength: namely, $\beta=4$ and $\beta=100$.

Both the weak $(\beta=100)$ and strong field $(\beta=4)$ simulations show a comparable behavior with a substantial enhancement of the magnetic pressure at the leading edge of the cloud as a result of field-line stretching there. This confirms and extends previous two-dimensional results (Jones et al. 1996; Miniati et al. 1999a). The importance of field-line stretching to field amplification and cloud motion can now be fully appreciated, since the slipping of the field lines around the cloud (which was prevented in two-dimensional simulations by their geometry) turned out to be a minor effect in our three-dimensional simulations. In general, moving clouds are reshaped by the field lines draping around their bodies, generating elongated structures oriented perpendicular to both the background magnetic field and the flow velocity. This is also important in terms of cloud collisions because it means that if two clouds are approaching each other moving through the same large-scale magnetic field, their elongated, cylindrical bodies will tend to collide with the aligned longer axis. This helps validate previous two-dimensional MHD cloud collision calculations (Miniati et al. 1999b) and sets important constraints for future work on that subject.

The main result of this study is that contrary to two-dimensional geometry where fluid instabilities were prevented by the growth of a strong magnetic field, in three dimensions, they are instead considerably hastened by it. This was clearly shown in Figure 2 where the dramatic difference between the purely hydrodynamic case and the $\beta=4$ MHD case is attributed to the accelerated development of an R-T instability in the latter case. It was also shown that for $\beta=4$, the timescale for the cloud disruption is reduced by a factor of $\sim 2$.

This work is supported at the University of Minnesota by the NSF through grants AST 96-19438 and INT95-11654, by NASA grant NAG5-5055, and by the Minnesota Supercomputing Institute. Work by D. R. is supported in part by KOSEF through grant 981-0203-011-2.

\section{REFERENCES}

Bowyer, S., Lieu, R., \& Mittaz, J. P. D. 1998, in IAU Symp. 188, The Hot Universe, ed. K. Koyama, S. Kitamoto, \& M. Itoh (Dordrecht: Kluwer), 185

Chandrasekhar, S. 1961, Hydrodynamic and Hydromagnetic Stability (Oxford: Clarendon)

Dgani, R., \& Soker, N. 1998, ApJ, 499, 83

Fabian, A. C. 1997, Science, 275, 48

Jones, T. W., Kang, H., \& Tregillis, I. L. 1994, ApJ, 432, 194

Jones, T. W., Ryu, D., \& Tregillis, I. L. 1996, ApJ, 473, 365

Kane, J., Drake, R. P., \& Remington, B. A. 1999, ApJ, 511, 335

Klein, R. I., McKee, C. F., \& Colella, P. 1994, ApJ, 420, 213
Mac Low, M.-M., McKee, C. F., \& Klein, R. I. 1994, ApJ, 433, 757

Miniati, F., Jones, T. W., \& Ryu, D. 1999a, ApJ, 517, 242

Miniati, F., Ryu, D., Ferrara, A., \& Jones, T. W. 1999b, ApJ, 510, 726

Ryu, D., \& Jones, T. W. 1995, ApJ, 442, 228

Ryu, D., Jones, T. W., \& Frank, A. 1995, ApJ, 452, 785

Ryu, D., Miniati, F., Jones, T. W., \& Frank, A. 1998, ApJ, 509, 244

Stone, J. M., \& Norman, M. L. 1992, ApJ, 390, L17

Xu, J., \& Stone, J. M. 1995, ApJ, 454, 172

Yi, Y., Walker, R. J., Ogino, T., \& Brandt, J. C. 1996, J. Geophys. Res., 101, 27,585 